\title{
FINE NEEDLE ASPIRATION CYTOLOGY IN TUMOUR DIAGNOSIS
}

D. E Obaseki (MBBS, MWACP, FMCPath)

Consultant Histopathologist, Department of Histopathology, University of Benin

Teaching Hospital, Benin City, Nigeria

Correspondence:

Dr. D. E. Obaseki

Department of Histopathology

University of Benin teaching hospital

Benin City Nigeria

\section{INTRODUCTION}

Fine needle aspiration cytology (FNAC), a technique for obtaining cellular material for cytological examination and diagnosis using a 21gauge or smaller needle, is performed using a 5,10 , or $20 \mathrm{ml}$ syringe either freehand or using special syringe holders. It allows a minimally invasive, rapid diagnosis of tissue samples but does not preserve its histological architecture.

Although exfoliative cytology (i.e. Pap smear) is universally accepted and used in Nigeria, aspiration cytology has yet to be widely accepted as a method of microscopic diagnosis. These two techniques differ in their aim. Exfoliative cytology is used primarily to detect precancerous and cancerous conditions that are not yet clinically apparent, aspiration cytology is used to determine the nature of clinically detectable tumours.

The advantages of FNAC are innumerable and these include cost effectiveness, rapid reporting and bedside diagnosis, minimal physical and psychological discomfort, elimination of a two-stage procedure for diagnosis and treatment, active participation of the patient in treatment planning, and serving as a therapeutic procedure for evacuation of cystic lesions ${ }^{1,2}$ Unfortunately this technique is under-utilised in most centres in Nigeria, even when most of them have inadequate theatre facilities, limited operating time, and a heavy patient load culminating in long waiting lists for operative procedures such as open breast biopsies. This results in delay in definitive diagnosis of lesions and institution of appropriate early management, thus leading to increased morbidity and mortality rates in these patients ${ }^{3}$. Yet this rather simple and inexpensive diagnostic technique has been in use in other parts of the world since the 1920seighty years ago!

\section{THE ORIGINS OF FNAC}

The earliest report of a needle technique being employed to obtain material for microscopy was by Kun in 1847 who described a "new instrument for the diagnosis of tumours". There followed sporadic reports of this technique, championed mostly by clinicians including Leydon who in 1883 used needle aspiration to obtain cells to isolate pneumonic microorganisms and Greig and Gray who in1904 diagnosed trypanosomiasis in cervical lymph node aspirates from patients with sleeping sickness in Uganda ${ }^{4}$. Their findings were reported by a captain Bruce (later of Brucellosis fame) in a British Medical Journal memorandum in 1904. Afterwards there were other reports of a similar technique to puncture and diagnose lymph nodes infected by Leishmaniasis and secondary syphilis ${ }^{5}$. 
In the mid-1920s there were attempts in New York and Chicago to employ large needle (1.2-3.0mm) aspiration for a variety of sites ranging through lymph nodes, prostate and breast but over time the dimension of $1 \mathrm{~mm}$ or less has come to be accepted as the definition of 'thin' or 'fine' 5,6 .

A detailed and systematic study on FNAC was carried out in the late 1920s by Hayes Martin, a head and neck surgeon and James Ewing, the chief pathologist at the New York Memorial Hospital. Their experience comprising 2500 tumours annually was documented by Fred Stewart (a Histopathologist) who then enunciated the fundamental principles regarding the philosophy of aspiration biopsy and emphasized the need for close clinical and pathological co-operation. However, full confidence in the procedure was never achieved and this period coincided with a fierce controversy both in Great Britain and the USA over the reliability and risks of open biopsy in surgical practice, which clinicians feared would increase the risk of tumour spread. However, as their fears were laid to rest, the popularity of needle aspiration waned to such an extent that by the 1960s the technique was all but obsolete in the USA $^{7,8}$.

Interest in the procedure was resurrected by Europeans in the mid 1950s. In contrast to Martin and Stewart who used thicker calibre (18 gauge) needles, the European workers popularized the technique employing thin needles (22 gauge and higher) with an external diameter of $0.6 \mathrm{~mm}$ or less. This is the technique known today as the fine needle aspiration (FNA) cytology. Developments in Stockholm Karolinska Radiumhemmet Hospital in Sweden were of utmost and fundamental importance. Here, workers such as Sixten, Franzen, Sordenstrom, and Torsten Lowhagen in collaboration with Joseph Zajicek applied the requisite scientific rigour to define precise diagnostic criteria in a variety of conditions. The sheer volume, histopathological correlation, follow-up information, coupled with superbly illustrated publications provided an ethos within the medical profession to allow the technique free reign. Their practice invented a novel specialty of 'clinical cytologist' who would examine the patient, aspirate the lesion, prepare and read the slide and arrange subsequent onward referral. They thus provided a model for FNA services for the rest of the world such that it is now part of all sophisticated departments. 5,6

\section{INDICATIONS/USES}

Thanks to radiological guidance, there is practically no organ or structure that is now beyond the reach of the FNAC needle. Masses of deep intraabdominal organs like the pancreas, kidneys, Para-aortic lymph nodes and also lung tumours are all subject to FNA cytodiagnosis.

Without image guidance, FNACs of superficial masses of the breast, superficial lymph nodes, thyroid gland, salivary glands and cutaneous swellings are done.

The technique can be

- Diagnostic or

- Therapeutic e.g. evacuation of benign breast cysts

The major indication for FNA relates to diagnosis of either primary malignancy or metastatic disease. As a diagnostic tool for evaluating potentially malignant conditions FNAC has the following indications

- Initial evaluation before treatment and in prognostication

- Evaluation of recurrence following therapy

- Evaluation of metastasis 
BENEFITS OF FNAC

It is now generally agreed among medical professionals that FNAC should be the initial diagnostic procedure for palpable breast lesions.

The advantages of FNAC include

- Cost effectiveness ( simple and cheap)

- It has lower risk than surgical biopsy

- It is readily repeatable and useful for multifocal lesions

- Minimal physical and psychological discomfort for the patient.

- Rapid reporting and bedside diagnosis of neoplastic, hyperplastic, and inflammatory masses.

- Active participation of the patient in treatment planning and provides opportunity for fuller preoperative counselling.

- Elimination of a two stage procedure.

- Therapeutic procedure for the evacuation of cystic lesions.

- Allows cases to be prioritized when there is a waiting time for surgery

- Permits the diagnosis of some benign conditions for which there is no need for surgery.

- Renders unnecessary the need for excision biopsy in advanced disease, elderly patients, or in cases where the treatment is non-surgical, e.g. in neoadjuvant chemotherapy.

- It is a rapid means of confirmation of recurrence of previously treated malignancy without surgery ${ }^{2}$.

\section{LIMITATIONS}

- Sampling is scanty and histological architecture is lost thereby rendering impossible diagnosis based on histology
- Inflammatory, metaplastic or degenerative lesions may mimic malignancy

- Diagnosis is indefinite in some conditions such as follicular adenoma vs. carcinoma of the thyroid

- Samples taken may not be representative of the lesion

- Difficulty of cytological diagnosis in some conditions e.g. lymphomas ${ }^{9}$

\section{COMPLICATIONS}

Complications are few and seldom serious. The incidence of major complications reported is well below $1 \%$ and generally in the range of $0.05 \%{ }^{5}$. Serious complications include:

\section{HAEMORRHAGE}

Serious haemorrhages have only been reported after FNA of deep structures such as the lung, liver, and kidney. Bleeding from CT scan-guided FNA of retroperitoneal adenopathy is reported at less than $1 \%$ despite the proximity to great vessels. ${ }^{5}$

Haematoma formation is unpredictable but can be minimized by prolonged pressure following aspiration, but sometimes the bleeding occurs very rapidly even before the needle is withdrawn. This can obscure the lesion and make a second aspiration difficult or impossible. It may render mammograms difficult to interpret for the following two weeks; hence it is recommended that all imaging investigations should be completed before FNA

\section{PNEUMOTHORAX:}

This is a rare complication found mainly in women with small breasts, in medial lesions or when sampling axillary lymph nodes. Large pneumothoraces are obvious, but small ones may go undetected. Clues such as sharp pain, coughing, or a 
hiss of air on withdrawing the needle without evidence of air in the syringe may occur. ${ }^{10}$

\section{VASOVAGAL REACTIONS}

These range in severity from mild lightheadedness to syncope.

\section{SEEDING OF TUMOUR}

Despite the persistent fears of clinicians that 'needling' a malignancy may result in dissemination, there is no evidence that this occurs with breast FNA $^{11}$.

\section{PAIN}

This is common on FNA but is transitory and is not usually severe. Aspiration from painful areas of benign breast change is sometimes associated with some pain. Carcinomas, particularly those with abundant fibroelastotic stroma, are often painful and this can be a guide to the aspirator that the needle has hit the lesion.

\section{PITFALLS IN FNAC}

Pitfalls are common in all aspects of diagnostic FNAC. There are many different kinds of pitfalls and they occur in many different contexts. Some pitfalls are the result of factors related to the sampling procedure. Others are created by unusual microarchitectural features or by the appearance of the cellular or stromal components specific to the lesion being investigated. 9,12

Errors in diagnosis of lesions can lead to over-treatment or delay in diagnosis and treatment. Aspiration cytology requires highly skilled and trained personnel in both aspiration and assessment. Stewart commented in 1933 that "until the pathologist has familiarized himself with the various pitfalls, errors are certain to occur" and "it must not be inferred that the diagnosis is always simple and that no errors have been made"7. For this reason, FNAC must always be interpreted in association with clinical and radiological opinions in the triple approach. The NHSBSP Cytology Guidelines also state that "under no circumstances should a cytological opinion of malignancy in the absence of mammographic and / or clinical evidence of malignancy be taken as authority for therapeutic surgery"13.

Lack of or misleading clinical information, non-representative samples, contamination of samples by tissue adjacent to the target lesion, artefacts caused by poor processing of samples and too much reliance on and technical failure of ancillary tests are all 'extrinsic' factors which can contribute to diagnostic errors in the interpretation in either of FNAC samples ${ }^{9}$.

'Intrinsic' factors which can lead to diagnostic pitfalls result in false positive or false negative diagnosis arise mainly due to deviations from general cytodiagnostic criteria which can occur in a variety of nonneoplastic and neoplastic lesions in different sites. The deviations may concern the microarchitectural pattern, the cellular composition or the stromal component of the target tissue.

Pitfalls are an inseparable part of the practice of FNAC, but its occurrence can be minimized if the requisite diagnostic rigours are applied and care taken to correlate cytology with clinical history and with clinical and radiological findings. Multiple sampling used liberally when indicated, independent double checking by two pathologists of all primary malignant diagnoses and most importantly experience acquired from frequent usage of this technique help to minimize the incidence of diagnostic pitfalls in FNA cytodiagnosis. 
FNAC IN UBTH

The popularity of FNAC in UBTH has been on the increase since the procedure was introduced to the hospital in the early 2000s. But it was been used rather sparingly as attested to by the fact that for the whole of the year 2003 only 5 FNACs were performed (all for breast masses). Most of which were for cases that were obviously clinically malignant. The surgeons were in these cases, for medico-legal reasons, looking for pathological justification to perform therapeutic surgery without necessarily subjecting the patient to two surgeries. The reasons for this initial apathy are manifold but were mostly centred on the reliability of the test result, the added cost implication to the patients and the timeliness of the result. After some of these fears and reservations were allayed, the procedure has gained currency among surgeons in UBTH to the extent that in the year 2005 the number of FNAC done for breast masses alone had risen to 103.
This has led to the setting up of a dedicated FNAC clinic in the department of pathology. In addition to breast lumps, other sites frequently aspirated in the clinic includes superficial lymph node enlargement, thyroid gland masses and salivary gland masses.

In breast aspirates, where the most experience has been acquired over the past 3-4 years, we have been able to demonstrate that our results are most reliable. In the period between January 2005 and March 2006 absolute and complete sensitivity of $84.6 \%$ and $97.4 \%$ respectively were achieved. The specificity was $64 \%$, positive predictive value for malignancies was $100 \%$, and there was an inadequate rate of $19.4 \% .^{15}$

These results are comparable to those obtained from other centres in Nigeria and abroad and well above the minimum standard of practice recommended by the National Health Service Breast Screening Programme (NHSBSP) guidelines of the United Kingdom.

Photomicrograph of a malignant breast smear done at the FNAC clinic in UBTH. (Haematoxylin \& Eosin X40).

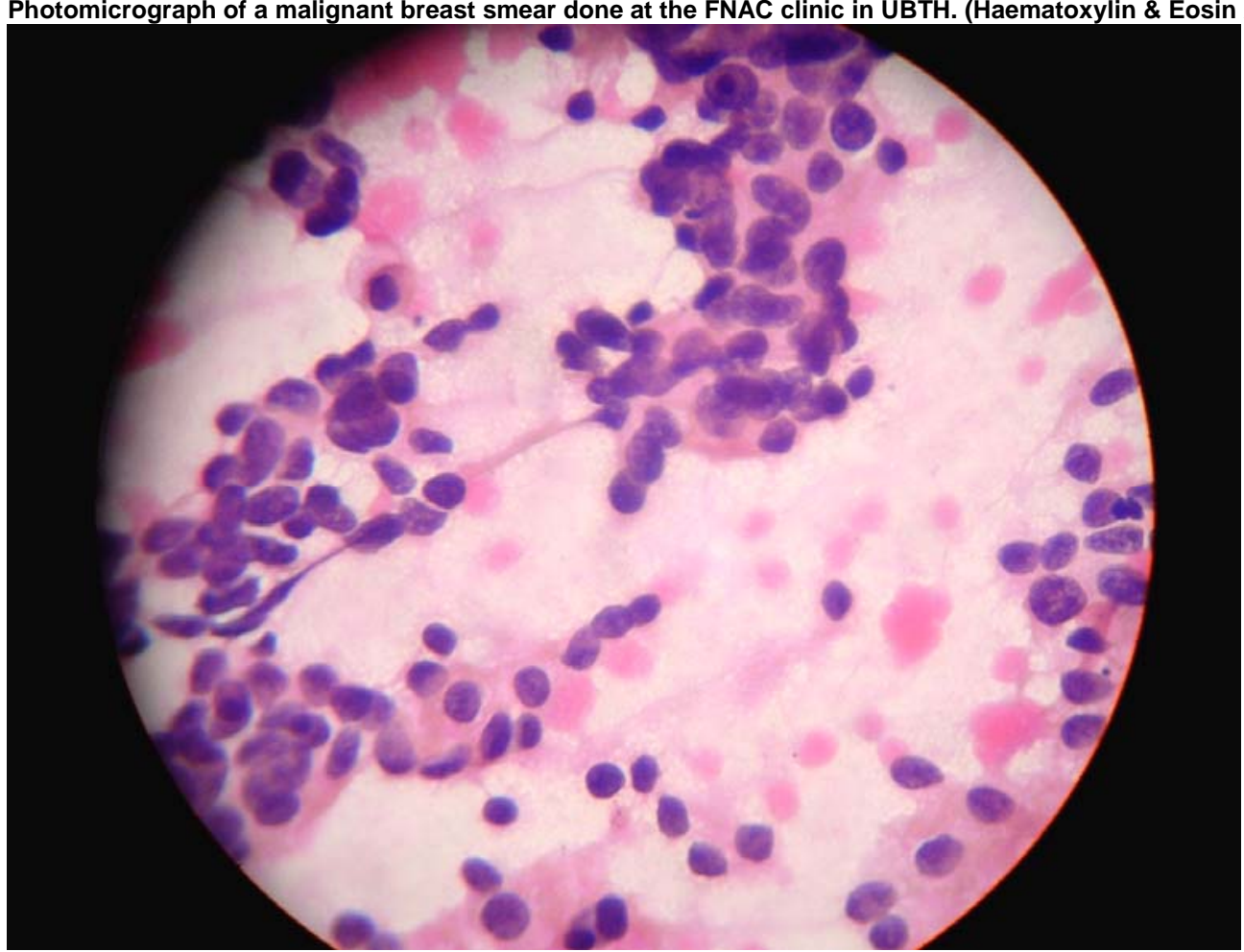


In conclusion, it should be said that FNA technique is simple but not banal. It requires a certain manual dexterity in the same way as surgical procedures do. Though it has now been established as the first line investigation of mass lesions wherever they occur in the body, it will continue to flourish only if used judiciously with realistic approach of what is achievable kept in view and clear communication between clinician and cytopathologist maintained.

\section{REFERENCES}

1. Fletcher SW, Black W, Harris R, Rimer BK, Shapiro S. Report of international workshop on screening for breast cancer. J Natl Cancer Inst. 1993; 85:16441656.

2. Vargas $\mathrm{HI}$ Masood $\mathrm{S}$, Implementation of a minimally invasive breast biopsy program in countries with limited resources. Breast J 2003;9 (Suppl 2):S8185.

3. Thomas JO, Amanguno AU, Adeyi OA, Adesina AO. Fine needle aspiration in the management of palpable masses in Ibadan: Impact on the cast of care. Cytopathology 1999; 10:206-210.

4. Webb AJ. Early microscopy: history of fine needle aspiration (FNA) with particular reference to goitres. Cytopatology 2001; 12:17.

5. Frable WJ. Needle aspiration biopsy: past, presnt and future. Hum Pathol 1989; 20:504-517.
6. Ansari NA, Derias NW. Fine needle aspiration cytology. J Clin Pathol 1997; 50:541-543.

7. Stewart FW. The diagnosis of tumour by aspiration. Am J Pathol 1933; 9: 801-812.

8. Martin HE, Ellis EB. Biopsy by needle puncture and aspiration. Ann Surg 1930; 92:169-181.

9. Orell SR. Pitfalls in fine needle aspiration cytology. Cytopatology 2003; 14:173-182.

10. Stevenson J, James AS, Johnston MA, Anderson IW. Pneumothorax after fine needle aspiration of the breast. BMJ 1991; 303:924.

11. Howat A, Coghill S. Normal breast cytology and breast screening In: Gray W, Mckee GT (eds). Diagnostic cytopathology. Churchill livingstone, Philadelphia 2003: 237-255.

12. Thunnissen FBJM, Kroese $\mathrm{AH}$, Ambergen $\mathrm{AW}$ et al. Which cytological criteria are the most discriminative to distinguish carcinoma, lymphoma, and soft tissue sarcoma? A probabilistic approach. Diag Cytopathol 1997; 17: 333-338.

13. Non-operative diagnosis Subgroup of National Coordinating Group for Breast Screening Pathology. Guidelines for Non-operative Diagnostic Procedures and Reporting Breast Cancer Screening. Sheffield, NHS Cancer screening 
programme, 2001 (NHSBSP NO. 50)

14. Lamb J, Anderson TJ. Influence of cancer histology on the success of fine needle aspiration cytology of the breast. J Clin Pathol 1989; 42:733-735
15. Obaseki DE, Olu-Eddo AN, Ogunbiyi JO. The diagnostic accuracy of fine needle aspiration of palpable breast masses in Benin City, Nigeria. WAJM (submitted). 